Mol. Cryst. Liq. Cryst., Vol. 450, pp. 97/[297]-104/[304], 2006

Copyright (C) Taylor \& Francis Group, LLC

ISSN: $1542-1406$ print/1563-5287 online

DOI: $10.1080 / 15421400600587837$

\title{
Magneto-Optic Tweezers Studies of Interactions in Liquid Crystal Colloids
}

\section{Jurij Kotar}

Dušan Babič

Faculty of Mathematics and Physics, University of Ljubljana, Jadranska, Ljubljana, Slovenia

\section{Mojca Vilfan}

J. Stefan Institute, Jamova, Ljubljana, Slovenia

\section{Martin Čopič}

Igor Poberaj

Faculty of Mathematics and Physics, University of Ljubljana,

Jadranska, Ljubljana, Slovenia and J. Stefan Institute, Jamova, Ljubljana, Slovenia

\begin{abstract}
Magneto-optical tweezers have been constructed and used for studying interactions between colloids in nematic liquid crystals. We used super-paramagnetic beads which induced planar anchoring at the surface and were suitable for optical trapping as well as manipulation by external magnetic field. Depending on the thickness of the sample, attractive or repulsive forces between two immersed spheres were observed: repulsive for sample thickness comparable to the bead size, and attractive in thick samples. This behaviour corresponds to quadrupolar interaction between two beads.
\end{abstract}

Keywords: colloids; interactions; liquid crystal; magneto-optic tweezers; optical tweezers

\section{INTRODUCTION}

Dispersions of colloidal particles in nematic liquid crystals (LCs) recently attract considerable scientific interest. One of the reasons is the long range of the interactions between the particles, mainly due

Address correspondence to Igor Poberaj, Department of Physics, University of Ljubljana, Jadranska 19, Ljubljana, SI-1000, Slovenia. E-mail: igor.poberaj@fmf.uni-lj.si 
to the orientational order of the liquid crystalline phase. The interactions are mediated by the elastic distortions of the director in the host material, depending on the director configuration in the sample and especially around the immersed particles.

When studying the inter-particle interactions, a very convenient way is to use a non-contact particle manipulation method in order not to influence the measurement. For this purpose, optical tweezers can be used. Ashkin [1] determined that micrometer-sized dielectric beads with a refractive index larger than the index of the surrounding medium can be trapped and manipulated by a strongly focused laser beam. We have shown, however, that in a nematic liquid crystal also a low-index particle $\left(\mathrm{n}_{\text {particle }}<\mathrm{n}_{\text {medium }}\right)$ can be trapped [2]. Two responsible mechanisms have been identified: at low trapping laser intensities the particle is trapped via its birefringent cloud, whereas for high powers, the reason for trapping is the laser-induced distortion of the director field. Experiments using LC as a host material have already been performed, mainly for determination of interaction forces between colloids [3,4] and determination of viscosity parameters [5].

Although many improvements can be made to reduce the influence of the laser light on the liquid crystal orientation, e.g. lowering the beam intensity, or use of a LC with small dielectric anisotropy, the effects of light on the director field can never be completely excluded. These effects influence the obtained results and make the interpretation of the observed phenomena more complex. Furthermore, optical tweezers are often too weak to overcome the inter-particle forces at smaller distances.

In order to avoid the disadvantages of the optical tweezers, we built magneto-optical tweezers. The setup is suitable for studying the interparticle interactions in undistorted samples as the magnetic field required to trap and manipulate a super-paramagnetic bead is small (order of $10 \mathrm{mT}$ ) and therefore does not influence the director configuration in the sample. We report on the first successful implementation of the magneto-optical tweezers setup for manipulating superparamagnetic beads in nematic liquid crystal.

\section{MAGNETO-OPTIC TWEEZERS}

Magneto-optic tweezers used in our experiments were laser tweezers and magnetic tweezers combined in a single instrument. The schematic of the experimental setup is shown in Figure 1. The setup was built around an inverted optical microscope (ZEISS, Axiovert 200M). Optical trap was generated with a single mode (TEM00) diode pumped $\mathrm{Nd}$ :YAG laser operating at $1064 \mathrm{~nm}$ (COHERENT, Compass 2500MN). 


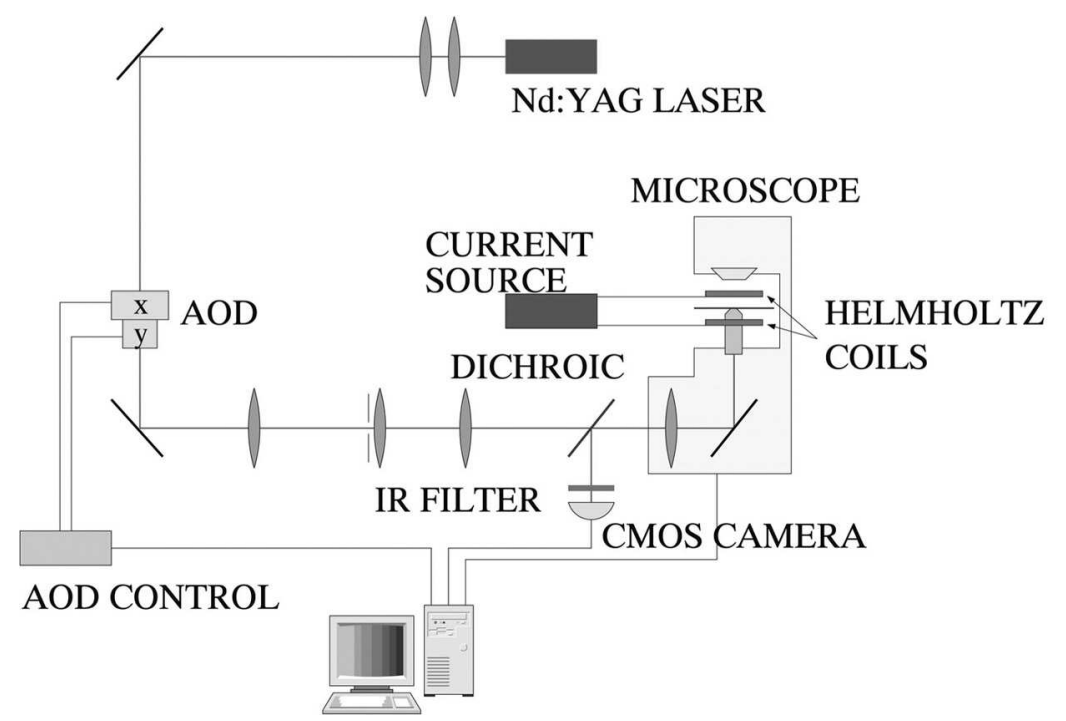

FIGURE 1 Schematics of magneto-optic tweezers setup. AOD - a pair of acousto-optic deflectors.

Infrared wavelength was chosen for its low absorption in liquid crystal and in colloidal particles. Laser beam diameter was expanded with a beam expander to match the size of the entrance pupil of a pair of acousto-optic deflectors (AOD), which deflected the beam in two orthogonal directions. The acousto-optic deflectors (INTRAACTION, DTD-274HA6) were used for precise laser beam steering and multiple trap generation. AOD combined with suitable driver electronics and software offered very high position and intensity accuracy of the trapping beam and were capable of generating several hundred traps in the sample plane. A set of relay mirrors and lenses was used for imaging the pivot point of AOD to the entrance pupil of a high numerical aperture water immersion microscope objective (ZEISS, Achroplan $63 / 0.9 \mathrm{~W}$ ). Samples were illuminated by a halogen lamp and observed in a bright field. Image acquisition was done with a fast CMOS camera (PIXELINK, PL-A741). The experiment was computer controlled by an integrated package of image acquisition and laser position control software and AOD control electronics (ARESIS, TweezTek 70).

The physical principle of magnetic tweezers relies upon the interaction between induced magnetic dipole moments in superparamagnetic beads. Magnetic interaction between magnetized colloidal particles was adjusted with a homogeneous external magnetic 
field generated by a pair of Helmholtz coils. Coils were driven by a precision low noise current source and the direction of the magnetic field was perpendicular to the sample plane. The magnetic field of around $30 \mathrm{mT}$ was high enough to completely saturate the magnetization of super-paramagnetic particles and thus resulted in the maximum possible interaction strength between the colloids used in our experiments.

\section{SAMPLE PREPARATION}

The schematic cross section of a sample cell with a colloid in a liquid crystal is shown in Figure 2a. Sample cells were made of a microscope and a cover glass glued together with a UV curing glue (NORLAND). The thickness of a cell was controlled by inserting a spacer of a known thickness between the glass plates. The cell thickness was later verified with a spectrophotometer (HP, UV-Vis 8453). The glass surfaces were carefully cleaned and treated with silane (ALDRICH $435708,0.5 \%$ solution in water) to ensure homeotropic alignment of liquid crystal molecules at the cell walls. The polymeric surface of the spheres induced planar alignment of liquid crystal molecules. Beads used in the experiments were super-paramagnetic spheres with a diameter of $2.8 \mu \mathrm{m}$ (DYNALBIOTECH, Dynabeads M-270 Epoxy) and $4.5 \mu \mathrm{m}$ (DYNALBIOTECH, Dynabeads M-450 Epoxy). Dried beads were suspended in nematic liquid crystal TL205 (LICRILITE by Merck, $\mathrm{T}_{\mathrm{NI}}=87^{\circ} \mathrm{C}$ ) or $5 \mathrm{CB}$ (Aldrich, $\mathrm{T}_{\mathrm{NI}}=35.1^{\circ} \mathrm{C}$ ) using a shaker and ultrasound bath. The cells were filled with freshly prepared mixture to avoid bead aggregation. Measurements were performed at room temperature.

(a)

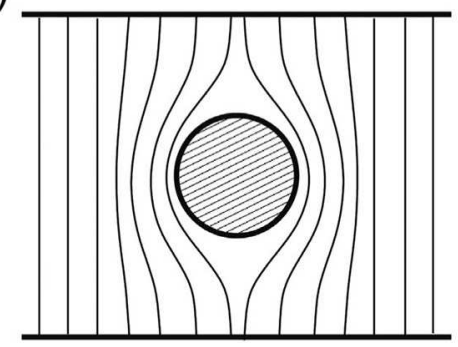

(b)

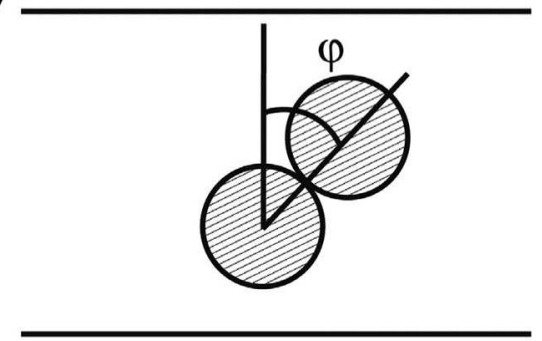

FIGURE 2 Sample cross section. (a) Silane treated glass plates ensured homeotropic alignment of liquid crystal, whereas the surface of the superparamagnetic colloid induced planar alignment. (b) In thick samples, the forces between the beads are attractive and the parameter $\varphi$ is introduced. 


\section{EXPERIMENT}

At the beginning of each measurement, two spheres were brought into vicinity (within a few micrometers) of each other with the help of optical tweezers (Fig. 3). The tweezers were then switched off and the behaviour of the now free beads was observed. The motion of the particles was recorded as a function of the applied external magnetic field. Even in the absence of the applied field, interesting phenomena was observed as qualitatively different behaviour of the motion occurred depending on the cell thickness.

In thin samples, where the thickness was less than approximately two bead diameters, the motion of the spheres was confined to two dimensions. In this case, the forces between the immersed beads were found to be repulsive. A typical spatial evolution of the distance between the two beads is shown in Figure 4 (black curve). If external magnetic field was switched on, the separation increased more rapidly (grey curve, $\mathrm{B}=3 \mathrm{mT}$ ). This is due to the fact that the magnetic field induced magnetic dipole moments in the spheres. As these dipole

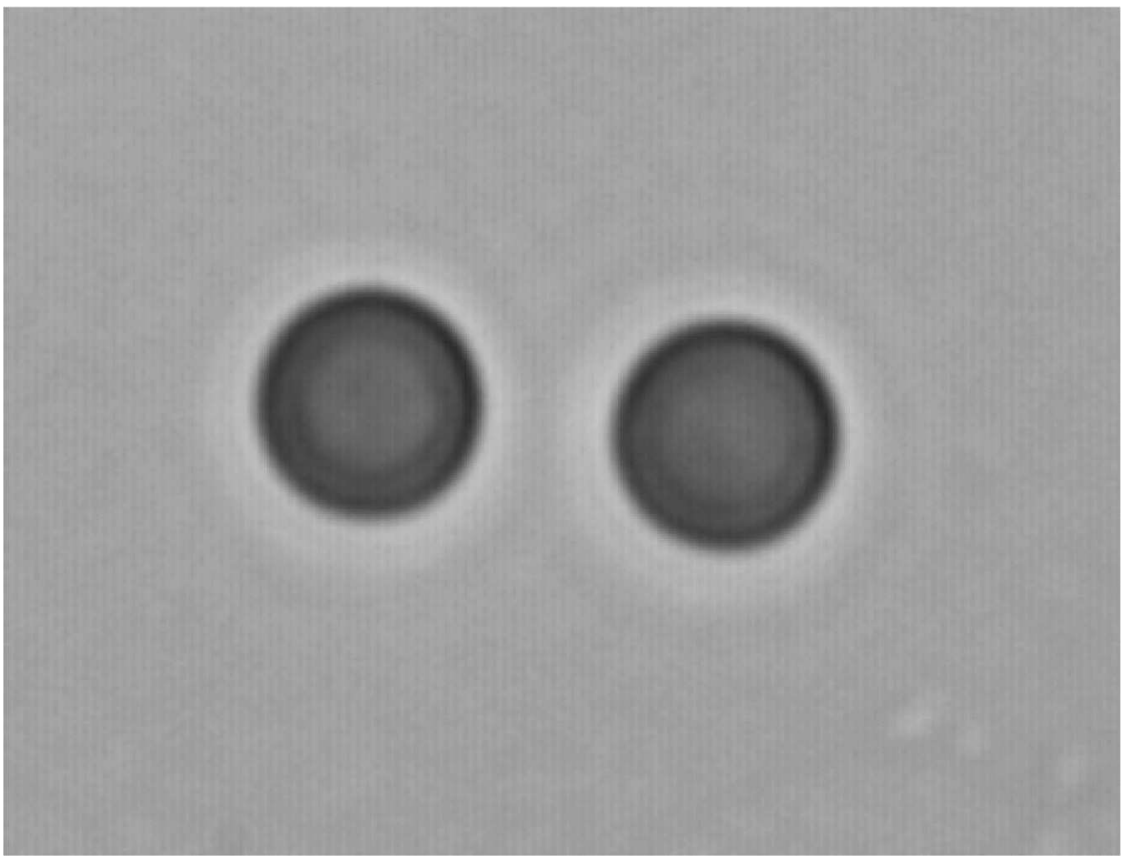

FIGURE 3 Two beads immersed in the liquid crystal. They were brought together using optical tweezers and released. Bead diameter is $d=4.5 \mu \mathrm{m}$. 


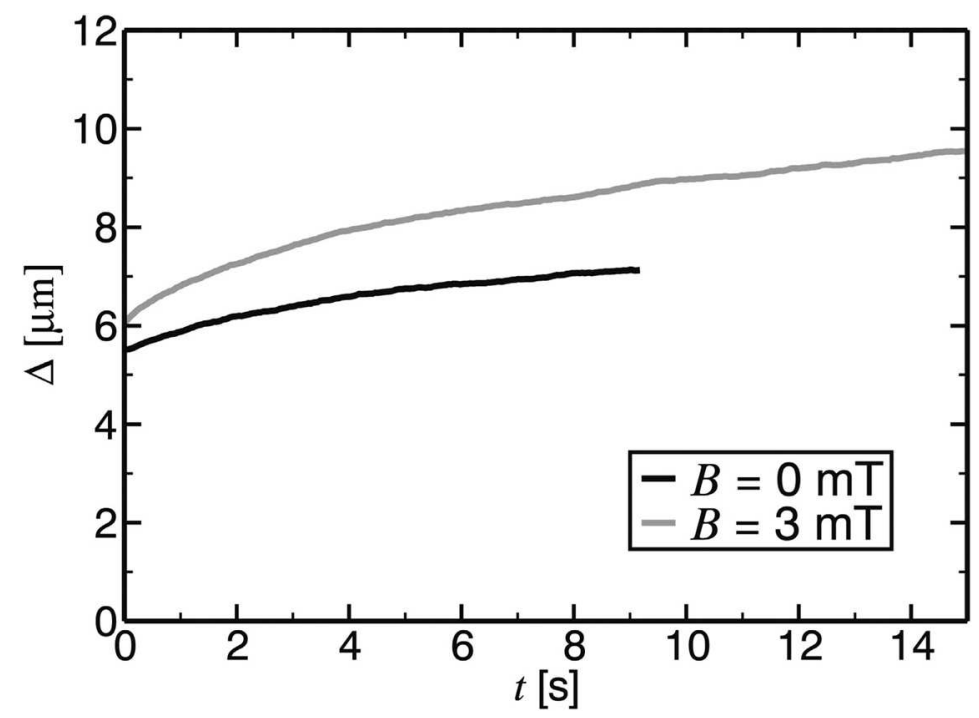

FIGURE 4 Distance $\Delta$ between two beads in a thin sample as a function of time $t$. The applied external magnetic field $B$ accelerated the separation.

moments were oriented parallel to the magnetic field, their magnetic dipole force was repulsive. The additional repulsive force thus depended on the strength of the applied magnetic field. If the initial distance between the beads was less than approximately 1 micrometer, the force between the particles was attractive due to structural forces in the liquid crystal. Even strong magnetic fields $(60 \mathrm{mT})$ were not sufficient to overcome the attractive force and the particles remained clustered.

In thick samples, the beads could move in all three dimensions. In contrast to thin samples, no repulsive behaviour was observed for any initial separation. With no external field applied, the spheres stuck together at a certain angle. For quantitative analysis we introduced $\varphi$ as the angle between the centres of the spheres and the average director (see Fig. 2b). Assuming that the spheres were in contact, horizontal distance $a$ between the spheres' centres was measured and angle $\varphi$ was calculated using the formula $\varphi=\arcsin (a / d)$. Angle $\varphi$ was found the same for all pairs of beads observed and was $\varphi \sim 37^{\circ}$ (Fig. 5). When external magnetic field - which was parallel to the average director - was applied, magnetic dipole moments in the beads were induced. Therefore in the presence of the field the beads tended to align on top of each other, i.e., $\varphi \rightarrow 0$. We measured the alignment 


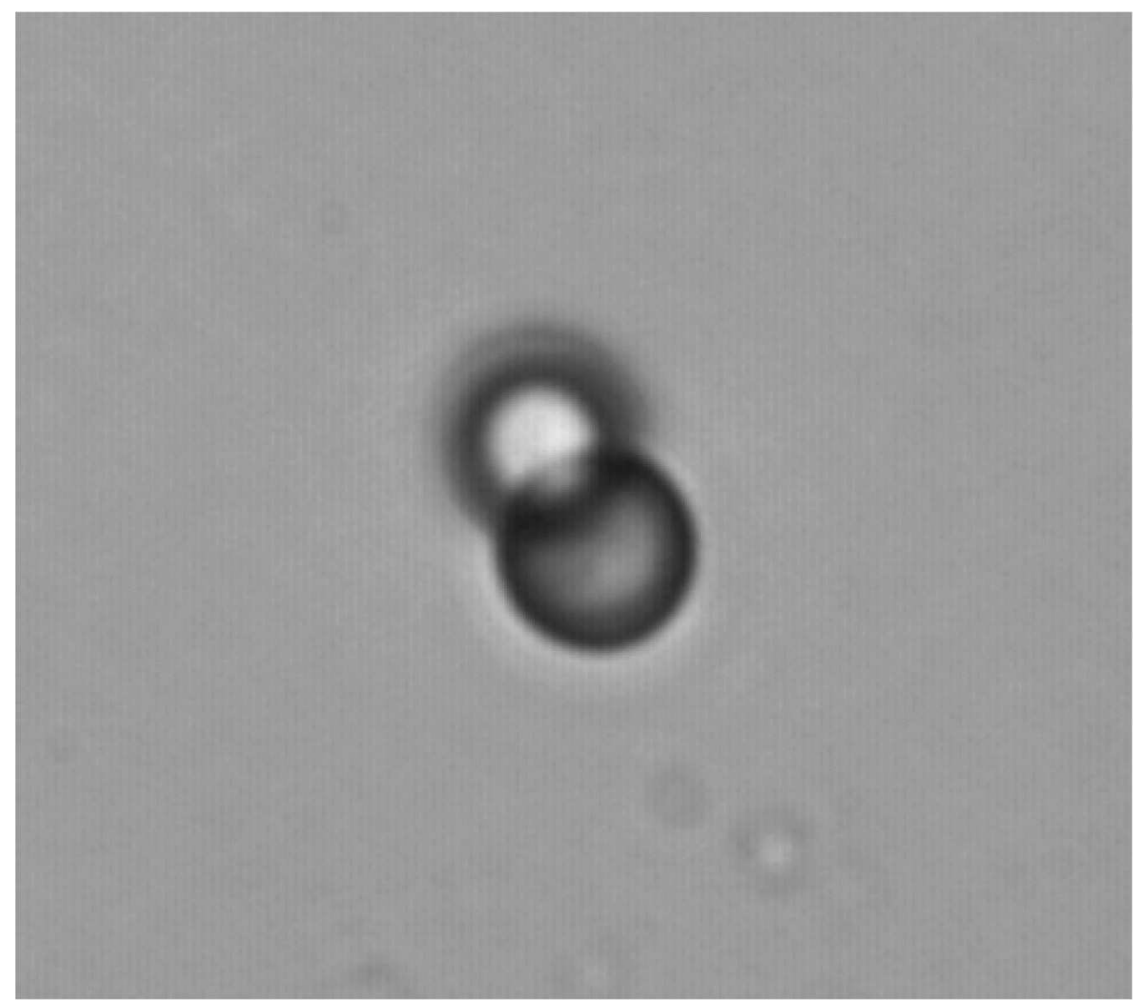

FIGURE 5 The interaction between two beads in thick samples is attractive and the beads form clusters. This is a top view and thus one bead is off focus. Bead diameter is $d=4.5 \mu \mathrm{m}$.

angle $\varphi$ as a function of the magnetic field and $\varphi$ decreased with increasing field as shown in Figure 6. The saturation equilibrium value was found to be less than $\varphi \sim 10^{\circ}$.

\section{DISCUSSION}

We have used magneto-optical tweezers for manipulating particles in nematic liquid crystals 5CB and TL205. Depending on the sample thickness, both attractive and repulsive forces between two immersed beads were observed, depending on the initial separation. In thin samples the forces at an initial separation greater than $\sim 1 \mu \mathrm{m}$ are repulsive and in thick samples attractive, which indicates that the beads act as quadrupoles. This is also expected due to planar ordering of LC on the bead [6]. When applying external magnetic field, 


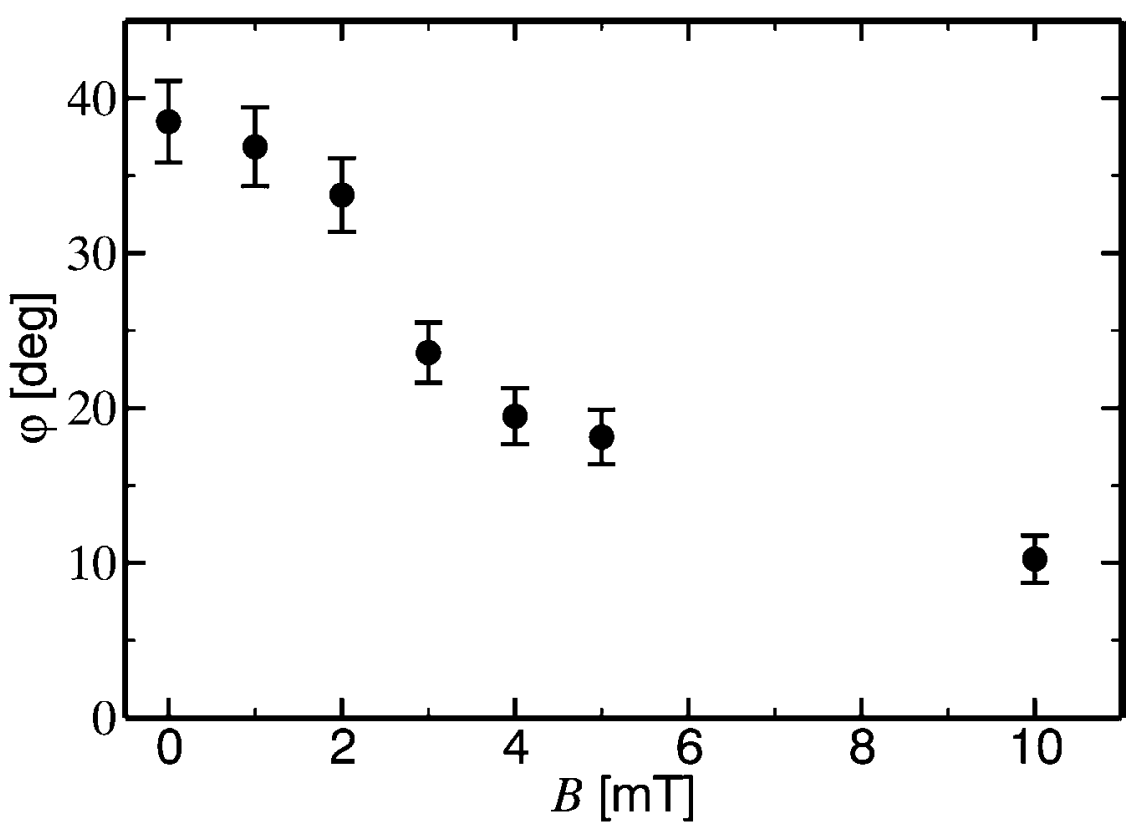

FIGURE 6 The alignment angle $\varphi$ versus external magnetic field B.

magnetic dipole moments were induced in the spheres. The alignment angle $\varphi$ was determined by equilibrium of the magnetic torque and the torque induced by the liquid crystal. The angle $\varphi$ decreased with increasing magnetic field and retained a finite value for large magnetic fields due to magnetization saturation in the spheres. Further experiments using magneto-optic tweezers have to be performed and they will ensure reliable analysis of the bead motion and inter-particle interactions as well as determination of viscosity coefficients without the distorting laser light.

\section{REFERENCES}

[1] Ashkin, A. (1970). Phys. Rev. Lett., 24, 156.

[2] Muševič, I., Škarabot, M., Babič, D., Osterman, N., Poberaj, I., Nazarenko, V., \& Nych, A. (2004). Phys. Rev. Lett., 93, 187801.

[3] Smalyukh, I. I., Kuzmin, A. N., Kachynski, A. V., Prasad, P. N., \& Lavrentovich, O. D. (2005). Appl. Phys. Lett., 86, 021913.

[4] Yada, M., Yamamoto, J., \& Yokoyama, H. (2004). Phys. Rev. Lett., 92, 185501.

[5] Gleeson, H. (2005). 8th European Conference on Liquid Crystals, Sesto (Italy), Invited Talk.

[6] Poulin, P. \& Weitz, D. A. (1998). Phys. Rev. E., 57, 626. 